\title{
EDUCACIÓN, DESARROLLO Y MODERNIZACIÓN ${ }^{1}$
}

\author{
Carlos Ruiz Schneider*
}

Según lo que se nos dice en trabajos recientes sobre historia de la educación chilena, la Reforma Educacional emprendida durante el Gobierno de Eduardo Frei Montalva, en la década de los 60, significa un cambio decisivo en el sistema educacional chileno, después de las Reformas de 1928 y 1929, que habían establecido a grandes rasgos el marco o el modelo educacional vigente en nuestro país hasta esa fecha ${ }^{2}$.

Creo que es posible mostrar que, en diversos sentidos, el modelo educacional de esta reforma constituye, a su vez, lo fundamental de la teoría educacional vigente hasta por lo menos 1981, año en que la dictadura militar impone una nueva teoría menos comprehensiva, centrada en el tema de la privatización y el mercado. Los comienzos de los 90 nos traerán por último la propuesta de un nuevo paradigma, fundado en el tema del crecimiento con equidad que constituye, hasta donde puede verse, un intento ecléctico de conciliar un modelo centrado en el mercado, con algunos elementos correctores, provenientes de la intervención estatal.

Querría sostener en esta exposición que la Reforma Educacional del Gobierno Frei, que ha sido evaluada en general como una reforma de carácter democratizador ${ }^{3}$, tiene como marco global no la democracia sino el modelo social y político de las teorías del desarrollo y la modernización. Esto importa una funcionalización global de la educación al crecimiento económico del país, naturalmente sobre la base de la mantención de su estructura social, marginalizándose así otros componentes posibles de una teoría educacional como su contribución a la formación para la ciudadanía, el acceso a formas de saber no instrumental o la reducción de las desigualdades sociales.

El discurso pedagógico de la Reforma es, tal vez, su mayor logro, en términos de permanencia en el tiempo: todavía hoy no es claro que se hayan consolidado paradigmas alternativos. Sin embargo su base teórica es también muy discutible, basada como está en teorías conductistas del aprendizaje que dejan fuera toda dimensión de sentido en su comprensión de la educación.

* Académico del Departamento de Filosofía de la Facultad de Filosofía. Humanidades y Educación Universidad de Chile, Investigador del CERC, Coordinador del Área de Filosofía de la Universidad Andrés Bello.

${ }^{\prime}$ Este trabajo ha sido posible gracias al aporte de FONDECIT, en el marco de un proyecto de investigación en curso sobre "Teorias educacionales y modelos políticos en Chile: 1960-1990". Lna versión anterior fue presentada al Seminario Internacional sobre "La transformación económico-social y las ciencias sociales" organizada por CERC y la Facultad de Ciencias Sociales de la Universidad de Chile, en noviembre de 1993. Agradezco la importante contribución de los Ayudantes de Investigación Vasco Castillo y Catalina Ruiz al presente estudio.

${ }^{2}$ En su trabajo sobre Reformas Educacionales ocurridas en Latinoamérica en los últimos 50 años y orientaciones pedagógicas que las han sustentado. Estudio de base: Chile (Ministerio de Educación y Universidad Simón Bolívar, Caracas, Venezuela, 1978) Iván Núnez sostiene que "(el) modelo de la contrarreforma (de 1929), a pesar de rectificaciones transitorias o de alternativas de detalle, subsistió en sus líneas esenciales hasta 1965 y en algunos aspectos, hasta el presente. La estrategia de cambios graduales y parciales pasó a ser la dominante hasta esta misma fecha". (p. 81).

${ }^{3}$ Ver, sobre este punto. C. Cox, "Tres reformas educacionales en Chile" en R.Alvayay, Carlos Ruiz (Comp.) Democracia y Participación (Santiago: CERC-Melquíades, 1988). 
No es evidente que las teorías del desarrollo y la modernización impliquen necesariamente un modelo político democrático, pero si lo hacen, se trata de un modelo elitista y etnocéntrico que querría también evaluar en esta presentación. Para concluirla haré un esbozo de evaluación basado en los estudios de M. Walzer, H. Arendt y J. Ranciere que insisten desde diferentes perspectivas en una necesaria ruptura con una funcionalización excesiva de la educación respecto de la economía y la producción.

Como lo he dicho más arriba, la visión más generalizada sobre la Reforma Educacional de 1965 es que se trata de un cambio educacional con un profundo contenido democratizador. Esta es, por ejemplo, la percepción de especialistas como Cristián Cox y en cierta medida también de Iván Núñez. Ahora bien, mi propia hipótesis de trabajo es que este proceso no apunta primordialmente a una extensión de la democracia, sino que se funda en una concepción muy precisa y determinada de la educación, que uno puede relacionar a concepciones más globales sobre el desarrollo y más precisamente sobre el desarrollo económico, cuya relación con la democratización del sistema educacional chileno existe, pero claramente subordinada al tema del desarrollo. La base sociológica de las teorías del desarrollo es provista a nuestro entender por las teorías de la modernización, esto es por una teoría de la transición de una sociedad tradicional a una industrial a través de la creación de roles propios de una sociedad industrial en sociedades que carecen de estos roles, a fin de favorecer este tránsito ${ }^{4}$.

La obtención de la meta del desarrollo económico supone un lugar esencial para la educación. Esto tiene su origen en estudios paralelos sobre el valor económico de la educación, debidos especialmente a T. Schultz, de la Universidad de Chicago, los que concluyen en que se debe comprender a la educación como inversión y como inversión en capital humano, esto es como una de las inversiones más rentables para el desarrollo económico de un país.

Esta visión de la educación como inversión es central en la evolución de las mismas teorías del desarrollo en la década de los 60. Por último, a estas concepciones se agrega la idea de que la obtención del desarrollo económico y la expansión de la industrialización suponen también un lugar fundamental para la planificación de la vida económica, ya que las fuerzas del mercado no son por sí solas capaces de producirlos. La planificación de la vida económica tiene por último como corolario el planeamiento integral de la educación a la que se asigna precisamente como función esencial adecuar la producción de profesionales y la formación técnica a las necesidades del desarrollo e industrialización. Estas nuevas tendencias, que otorgan un lugar creciente a los estudios económicos sobre la educación, no van a tener ya como origen los trabajos de intelectuales o educadores

\footnotetext{
${ }^{4}$ Es importante hacer notar en este punto que las teorías de la modernización otorgan un valor central en la estructura social buscada, esto es en una sociedad industrial, a la empresa, la eficiencia productiva y a los empresarios. En David Apter, por ejemplo, uno de sus representantes más destacados, los índices principales de modernización son los siguientes: roles profesionales y empresariales e ingresos per cápita y tecnología. (Apter, 1972, 73) Para Seymour Martin Lipset, la formación de aptitudes empresariales es la función modernizadora fundamental que deberían cumplir los sistemas educacionales latinoamericanos (Lipset, 1967). Además del privilegio a la empresa y a los empresarios, estos autores son también buenos ejemplos del caráter apolítico y "acultural", para emplear una expresión de Charles Taylor, de la mayoría de las teorías de la modernización.
} 
nacionales. Este origen proviene indiscutiblemente y sobre todo de lo que podría llamarse el campo educacional internacional: la UNESCO, la OEA, la Comunidad Económica Europea, etc., a la que se pliega la alta burocracia estatal.

Una expresión de indudable importancia de lo que acabo de decir es la Conferencia sobre Educación y Desarrollo Económico y Social en América Latina de marzo de 1962. Esta Conferencia sigue a una reunión previa en Punta del Este en agosto de 1961 en donde se lanzó la Alianza para el Progreso - significativamente dos años después del triunfo de la Revolución Cubana- la que trazó un plan de 10 años para el desarrollo de la educación, en un plan de contención de los movimientos de izquierda en el continente. Como podrá verse en lo que sigue, los temas centrales de esta Conferencia van a coincidir en lo esencial con las ideas centrales de la Reforma Educacional de Frei.

En el Discurso Inaugural de la Conferencia, organizada por CEPAL, UNESCO, OIT, OEA y FAO, el Secretario Ejecutivo de CEPAL, Raúl Prebisch sostenía que "...la desproporción entre recursos externos e internos y las necesidades de inversión impone en forma ineludible la necesidad de planificación en América Latina. Planificar implica establecer un orden de prelación en esas inversiones. Y como no nos es dado hacer todas éstas en forma simultánea, es natural que en un orden de prelación corresponda a las inversiones en educación un lugar destacado, subrayando que se trata de inversiones y no gastos. Pero en el mismo campo educacional se necesitan claras orientaciones y objetivos. Hay formas de educación - las de adiestramiento técnico- que van a contribuir directamente al aumento de la producción, y hay otras que sólo podrán abordarse plenamente cuando el crecimiento del ingreso por habitante permita disponer de los recursos necesarios para hacerlo" (Prebisch: 1962, 3). Me parece que el texto de Prebisch es extremadamente claro en precisar la importancia que las nuevas orientaciones políticas en América Latina atribuyen a la educación. Pero podemos ver en él también inmediatamente sus limitaciones: desde la perspectiva del desarrollo — y es ésta la perspectiva que se va a transformar en dominante en las reformas que vienen - lo que interesa primordialmente en la educación es el adiestramiento técnico y la formación de mano de obra especializada.

En las investigaciones de Th. Schulz, que son la base de la idea de la educación como inversión, y a pesar de no reducirse la educación a sus elementos económicos, el punto es bastante claro. Para Schulz, el tipo de educación que hay que contar como inversión es sobre todo la enseñanza técnica. Según este autor, los trabajadores "se han convertido en capitalistas, no a causa de la difusión de la propiedad de las acciones de las compañías, como lo desearía la opinión popular, sino debido a la adquisición de conocimientos y técnicas que tienen un valor económico. Estos conocimientos y técnicas son en gran parte producto de una inversión y, combinados con otras inversiones humanas, explican de manera predominante la superioridad productiva de los países técnicamente avanzados". (Schulz: 1968, 135-136).

H. M. Phillips Director de la División de Análisis del Departamento de Ciencias Sociales de UNESCO reafirma, en un ensayo escrito en 1965 para un manual de esta institución sobre el planeamiento de la educación, la visión prevaleciente en este sentido, en especial para América Latina. Sostiene allí entre otras cosas que "hasta ahora pocos países han dado importancia, por lo menos en su política general, al papel de la educación en la formación de la mano de obra y de los recursos humanos en un sentido económico ! profesional. La educación se ha considerado sobre todo como una formación para la iida, más bien que como una formación para ganarse la vida. Ha habido interés en las necesidades educativas del niño para formar su personalidad, como futuro "ciudadano", 
sin preocuparse de su futura contribución al desarrollo económico y social, en sentido amplio. Esto no puede dar buenos resultados en las economías estacionarias o de lento desarrollo cuyo futuro depende de que logren romper el círculo vicioso de la pobreza y de un sistema social que impide el desarrollo. La mayor o menor importancia de la formación profesional o de la educación cívica varía según el nivel de desarrollo de la sociedad". (Phillips: 1965, 22). Como lo vemos en este texto, para los planificadores educacionales, en los países en desarrollo, las funciones políticas de la educación, en términos, por ejemplo, de una formación para un ejercicio más activo de la ciudadanía, tienen que subordinarse a la formación de la mano de obra y de recursos humanos.

En general, volviendo ahora a los textos de la Conferencia, que han sido muy bien analizados por Rodrigo Vera ${ }^{5}$, se piensa la educación sobre la base de dos ejes.

En primer lugar, como lo hemos dicho, como factor de desarrollo económico, como un medio técnico (inversión, planeamiento) de contribuir a este desarrollo.

Se constata en general, en los países desarrollados, "la tecnificación general de la existencia" y la educación es convocada a contribuir a la " adaptación a lo que esta técnica representa... y que se traduce sobre todo... en la necesidad de contar también con personas que posean una orientación tecnológica general de extraordinaria flexibilidad".

El valor económico de la educación es, como acabamos de decirlo, extraordinariamente destacado. La influencia de la educación "sobre la invención tecnológica, la difusión de innovaciones, la aptitud empresarial, los patrones de consumo, la propensión al ahorro, la adaptabilidad a cambios económicos y la participación activa de los distintos sectores sociales en las tareas del desarrollo" son algunos de estos aspectos del valor económico de la educación que la Conferencia subraya especialmente.

En segundo lugar, la educación es concebida como un medio de cambio social, de transformación social. Pero ¿qué es el cambio social desde el punto de vista de estas concepciones? Es básicamente un medio de selección y de ascenso social, de movilidad social, lo cual es otra exigencia del progreso técnico.

Estamos aquí en el centro del principio político de las teorías del desarrollo: el sistema educativo es necesariamente selectivo, "en él se filtran y se decantan los talentos de que dispone la sociedad en un momento dado para situarlos en aquellas posiciones en que puedan desplegar su máxima efectividad" (Boletín $\mathrm{N}^{\mathrm{o}} 14$, p. 59). Sin embargo, continúa el documento, "el proceso selectivo en vista del mérito realizado por la educación, rompe o quiebra los estratos tradicionales y abre las puertas a un rápido dinamismo en la movilidad social"(Ibid., p. 59). En otro tex to del mismo documento, la discusión de la Conferencia reafirma esta opción por las elites, característica por lo demás de las teorías de la modernización. Las elites, se nos dice en ese documento "seguirán existiendo, pero serán diferentes de las anteriores en su origen y función. Surgirán de todas las capas sociales y su acción irá encaminada a ayudar a las colectividades a buscar su camino y a construir un mundo nuevo de mutuo entendimiento y de paz para todos" (Ibid., p. 54).

Como puede verse las ideas dominantes de la Conferencia apuntan hacia dos orien-

\footnotetext{
${ }^{5}$ Para un análisis más detallado de la Conferencia véase, Rodrigo Vera, El provecto edacacional de la teoría del desarrollo (Análisis de la Conferencia sobre Edacación y Desarrollo Económico y Social en América Latina, 1962), (Santiago: PliE, 1985).
} 
taciones. La primera, que deriva de la relación fuerte entre educación y economía, marginaliza toda forma de conocimiento que no sea tecnológico o científico ${ }^{6}$.

La segunda orientación, centrada en el tema de las elites tiene aún más pertinencia para nuestro propósito. Creo que de lo expuesto se deriva claramente que la concepción del régimen político asociada a las teorias del desarrollo, pero sobre todo a las teorías de la modernización es una teoría elitista. Como lo sugerí antes, no es seguro que estemos aquí en presencia ni siquiera de una teoría elitista de la democracia, al estilo de Schumpeter, Lipset o Sartori. En la versión de Apter, por ejemplo, hay modernización por igual, dadas ciertas características en países como Chile, Nigeria, China o la Unión Soviética. Según este autor, en los países en vías de modernización han surgido sistemas políticos que califica como populistas o predemocráticos y que requieren simpatía y comprensión. A proximarnos a estas "sociedades como predemocráticas nos permite visualizar a ciertas instituciones de coerción como necesarias quizás a la organización e integración de una comunidad que se moderniza. Necesitamos confrontar la posibilidad de que las instituciones representativas pueden no funcionar en muchas sociedades en vías de modernización..." (Apter: 1965, 2-3). Como lo vemos en este texto la variable democrática no entra en estas teorías como una variable pertinente. En lo que toca al Tercer Mundo, las teorías del desarrollo y la modernización parecen implicar que la democracia como régimen político es algo demasiado refinado para nuestra región. En esto estas teorías no hacen sino continuar a otras visiones desarrollistas más antiguas, por ejemplo en los pensadores liberales del siglo XIX, que circunscribían en general la vigencia del liberalismo y la democracia a los países europeos y a los Estados Unidos. Para Lipset, por ejemplo, en un libro clásico Elites y desarrollo en América Latina, "nos interesamos en las elites por nuestra preocupación considerable por el desarrollo social, económico y político.Y aunque hay muchos factores que influyen en la tendencia al desarrollo de una nación, es evidente que, independientemente de las diferencias en los sistemas sociales, uno de los requisitos para el desarrollo es una elite competente que quiera modernizar su sociedad". (Lipset: 1967, 10). Según Lipset una de las tareas fundamentales de las sociedades que buscan la modernización, es precisamente la creación y formación de elites, fundamentalmente de elites empresariales innovadoras, a través de un sistema educacional modificado que privilegie orientaciones hacia la actividad productiva, la ciencia y la tecnología.

\footnotetext{
${ }^{6}$ Esta tendencia es más explícita en autores ligados a la teoría sociológica del desarrollo, como Gino Germani, por ejemplo. En su libro Política y sociedad en una época de transición. De la sociedad tradicional a la sociedad de masas, este autor sustiene que la secularización del conocimiento propio de una sociedad industrial exige que la "separación del conocimiento científico de la naturaleza, con respecto a toda actividad intelectual o forma de conocimiento (por ejemplo leológica, filosófica) debe marcarse de manera en extremo clara y producir consecuencias en el campo de la organización de la enseñanza y la investigación..."(p. 82). En este mismo sentido, Germani agrega que el proceso de secularización que caracteriza a la transición a la sociedad industrial requiere un "cambio en el contenido de la educación: se requiere, en efecto un fortísimo incremento de la enseñanza técnica y científica, y . como es sabido, este requerimiento suele chocar con los valores de prestigio que en las sociedades tradicionales se asignan las clases superiores y también con otras actividades y valores. especialmente religiosos... Si, en efecto, el surgimiento de una ciencia natural es una condición inmediatamente vinculada al desarrollo económico, la extensión de este tipo de conocimiento a la esfera de las acciones humanas es probablemente otra condición necesaria... El carácter menos inmediato de la vinculación y el hecho de que dicha esfera de conocimiento se halla mucho más interferida por valores de la sociedad tradicional puede explicar las mayores resistencias que se producen con respecto a la secularización del conocimiento en las ciencias del hombre. De todas maneras, históricamente, este proceso de secularización acompaña de manera estricta al desarrollo económico, y en particular los avances de la Sociología científica en todos los países en los últimos años han sido unánimemente reconocidos como una expresión de este proceso". (Ibid., p 91 ).
} 
Esto, de nuevo, es de una importancia crucial en América Latina ya que los valores latinoamericanos serían "antitéticos respecto de la lógica profunda de un sistema industrial en gran escala". (Lipset: 1967, 51).

Como era de esperarlo, en función de la idea de planificación y de planeamiento educacional, la Conferencia reafirma el rol fundamental que cabe al Estado en la integración de la educación al desarrollo. Según el Boletín $N^{\circ} 14$ de la Conferencia, hubo "unanimidad en que al Estado le corresponde desempeñar una función primordial en el dasarrollo de la educación y que gracias a ello todo miembro de la comunidad deberá tener derecho de acceso a las instituciones educativas sin discriminación alguna. El reconocimiento de ese principio no supone menosprecio ninguno al valioso aporte que en este campo ha prestado la iniciativa privada en la mayoría de los países. Sin embargo, para hacer efectivo aquel derecho, es sólo el Estado el que puede asegurar el carácter gratuito de la educación, por lo menos en la primaria, que sigue siendo la base insustituible de toda estructura nacional educativa orientada hacia el progreso" (p. 77-78).

$\mathrm{Al}$ interior del sistema educacional que la Conferencia describe en sus grandes rasgos, un aspecto de importancia es, por último, el que concierne al rol de los docentes. El análisis de los documentos nos muestra que, como lo subraya Rodrigo Vera, este status puede describirse como el de un "técnico calificado de mando medio", ubicado, en los niveles de decisión del sistema, por debajo de los planificadores y expertos de alto nivel técnico que incluyen a economistas, sociólogos y probablemente también psicólogos educacionales. No hay pues reconocimiento de la capacidad de los docentes para participar en los niveles directivos del sistema, aunque se enfatiza en cambio el rol más activo que cabe a los alumnos en el proceso de aprendizaje, esto siempre, claro está, al interior del marco fijado por los planificadores.

\section{II}

Sin duda, la influencia de las concepciones desarrollistas es fundamental en la ideología política de la Democracia Cristiana en el período del Gobierno de Frei. Probablemente ella sustituye al corporativismo original de algunos de los líderes de la Falange y tempera también la influencia anterior de Jacques Maritain. Según el historiador Mario Góngora, desde que sube a la Presidencia Eduardo Frei, "la política chilena toma un nuevo estilo, caracterizado por la influencia de los cientistas sociales y de los puntos de vista de la CEPAL en los políticos del partido gobernante". (Góngora: 1986, 249).

Para Alan Angell, las políticas sociales y económicas del período apuntaban, a diferencia de los Gobiernos anteriores, a la "estabilización mediante la reforma estructural, para así producir crecimiento económico y redistribución del ingreso". (Angell: 1993, 50). Con ello se esperaba contribuir a la gestación de una sociedad comunitaria que contrarrestara y marginalizara la fuerza de los partidos socialista y comunista en la sociedad chilena, a través de la integración al sistema imperante del grueso de los sectores populares no organizados que permanecían aún fuera del área de influencia de la izquierda. En este sentido, la política de Frei continúa una larga tradición de oposición al socialismo, constitutiva de la idea misma de desarrollo, desde la 2a post-guerra con el Punto IV de $\mathrm{H}$. Truman y que renueva en otras circunstancias la Alianza para el Progreso de Kennedy teniendo en la mira a la Revolución Cubana. ( H. Arndt, 1992).

Las principales reformas de estructura que Frei procura llevar a la práctica son la "chilenización" del cobre, la reforma agraria y la organización de los sectores populares 
urbanos y rurales. Según el mismo Angell, la reforma educacional se inscribe en este mismo plan de reformas de estructura.

En un discurso en el que pone en marcha la Reforma Educacional, en 1965, Frei parte por hacer un diagnóstico de la situación actual, en materia de educación, en América Latina. La situación de la región se caracteriza por la extensión del analfabetismo y por una baja escolaridad; por la escasez de la educación técnico-profesional y por una Educación Superior que sólo sirve a una minoría privilegiada. Lo anterior, señala Frei, "afecta al progreso social y económico. En lo social, no puede vivirse la auténtica democracia que sirve de ordenamiento a los pueblos hispanoamericanos. En lo económico, su consecuencia es la baja productividad..." (Frei: 1965, 16). Sin embargo, según Frei, en el último tiempo se ha comprendido "que la educación constituye una de las formas de capitalización de la riqueza de un país, expresada en los talentos del hombre" (Ibid.,16). Es por esto que, en la visión del político chileno, cada minuto que se pierde en "la puesta en marcha de una política educacional que incorpore el mayor número posible de personas en edad de recibir educación y que al mismo tiempo prepare en todas las especialidades profesionales que la producción exige, constituye una pérdida irreparable. Es un capital humano, el más valioso que puede poseer una nación, el que se frustra". (Ibid., 17). En esa misma ocasión, y al interior de esta perspectiva marcada por las teorías del capital humano y por los estudios sobre el valor económico de la educación, el Ministro de Educación de Frei, Juan Gómez Millas, recuerda en su discurso que la educación, tradicionalmente considerada un lujo y una forma de consumo, "hoy es estimada consumo - inversión. Como inversión es la que tiene un más alto rendimiento económico y su acertada administración es decisiva para todos los aspectos fundamentales del desarrollo nacional". (Ibid.,27).

Desde una perspectiva semejante, quien es, tal vez, el principal teórico de la Reforma, el Subsecretario Mario Leyton extrae también su visión de las relaciones entre educación y desarrollo, de las teorías de la modernización. En un documento de la Comisión de Planeamiento Integral de la Educación, publicado en 1967, sostiene que el proceso de transición que vive Chile "de una sociedad pre - industrial a otra de tipo industrial, debe ser debidamente considerado para definir una estructura educacional que responda a los requerimientos del desarrollo. Deberá tenerse presente, entre otros factores, la aceleración de los cambios tecnológicos y el continuo desplazamiento de técnicas y especialidades profesionales en sus diversos niveles". (Leyton: 1967, 60). En términos de una concepción más global, esta relación fuerte entre educación y desarrollo tiende a priorizar en el sistema educacional, el impulso a la educación técnica, a la ciencia y la tecnología. Esta primacía de la educación técnica deriva en realidad de dos raíces diferentes. La primera es, como lo hemos visto, la teoría del capital humano y la relación entre educación y recursos humanos. En su libro sobre La experiencia chilena: La Reforma Educacional 1965 - 1970, el mismo Mario Leyton subraya que "(el) gobierno actual tiene el convencimiento de que la educación técnico - profesional es uno de los factores esenciales para conducir al país hacia un desarrollo económico y social que descanse sobre bases sólidas, dado que el crecimiento de la economía está íntimamente relacionado con el aprovechamiento oportuno y eficiente de las aplicaciones de la ciencia y la tecnología en los diversos procesos productivos; es indispensable para lograr su óptimo aprovechamiento, que el país cuente con personal bien calificado en todos sus niveles". (Leyton: 1970, 82).

Una segunda fuente de la idea de priorizar la enseñanza técnica proviene, de nuevo, 
de las teorías de la modernización. Aquí el tema es la medida en que la educación latinoamericana se funda en, y difunde, valores hostiles al trabajo productivo. En un breve ensayo del mismo Mario Leyton y Antonio Carkovic sobre la reforma de la enseñanza media, ambos autores sostienen que las ciencias y la tecnología "enriquecen las dimensiones y virtualidades del humanismo contemporáneo. Los valores implícitos en aquellas áreas de nuestra cultura tienen un fuerte poder formativo. Opera aquí todo un cuadro axiológico nuevo que ha de ir desplazando la preponderancia de ciertos valores que distorsionan las posibilidades del desarrollo, subestimando el trabajo técnico y manual". (Leyton y Carkovic: 1968, 11).

Creo que este breve esbozo puede bastar para mostrar la medida en que el marco conceptual global de la Reforma Educacional de Frei depende también de las concepciones sobre el desarrollo. No se trata, por lo demás, de un hecho aislado ni original del gobierno demócrata cristiano en Chile. Como lo muestra James Bowen en su Historia de la Educación ${ }^{7}$, los modelos desarrollistas y la idea de planeamiento educacional conforman un modelo vigente prácticamente en todos los países occidentales a comienzos de los años 60 .

Ahora bien, querría esbozar, al final de estas páginas, una caracterización algo más precisa de los principios y consecuencias políticas que se derivan del modelo del desarrollo. Hemos mencionado ya algunas de esas consecuencias. Entre otras, hemos subrayado la idea de integración social como alternativa al modelo socialista o marxista de la lucha de clases. En segundo lugar hemos dicho que la Reforma de 1965 busca sustituir a las elites tradicionales y los cuadros dirigentes de la sociedad tradicional por nuevas elites de capas medias y en especial por empresarios, técnicos y científicos sociales, lo que importa una nueva economía de las relaciones de poder al interior de las escuelas y las Universidades y en general, al interior de la organización de la cultura nacional ${ }^{8}$. Hemos subrayado por último, que estos cambios se orientaban hacia la configuración de un nuevo elitismo, implícito en los análisis y las prescripciones de las teorías de la modernización. Sobre este último punto, como lo hemos observado también, las opiniones de los estudiosos de la Reforma de 1965 estan divididas, por lo que se impone la necesidad de un análisis más detallado.

Parece claro, en primer lugar, que el principio político orientador de la Reforma es el de igualdad de oportunidades.

La opción por este principio, que puede sin embargo articularse muy bien con una forma de elitismo, se funda, en los técnicos que dirigen la Reforma, en una cierta visión del sistema educacional chileno. Para Emesto Schiefelbein, por ejemplo, uno de los logros de la Reforma Educacional ha sido que "(los) dos sistemas paralelos (que existían antes, en los cuales se tenía diferente educación según los recursos económicos: o bien desde los liceos a las universidades, o bien desde las primarias a las Escuelas Normales o a las profesionales) se han integrado en un sistema nacional". (Schiefelbein: 1969, 183). En opinión de Mario Leyton, en una sociedad en vías de desarrollo, el sistema educacional debe impulsar el desarrollo económico y social y transmitir sistemas de valores favorables al cambio social. Ahora bien, la educación chilena, para este experto, "no

Véase sobre este punto, James Bowen, Historia de la Educación Occidental (Barcelona: Editorial Herder, 1985), Tomo III, Cap. XV

* Ver sobre esta orientación a la sustitución de las elites tradicionales, Juan Gómez Millas, "La formación de personal scentífico y técnico para el desarrollo nacional" en Revista de Educación, № 20, septiembre 1969, p. 26 y sgtes. 
había estado cumpliendo integralmente estas funciones. Esto se comprobaba por la desigualdad de oportunidades de educación...y la imposibilidad práctica de las familias de escasos recursos de costear la educación de sus hijos; y por la rigidez y falta de diversificación de los programas que se orientaban selectivamente hacia la Universidad. Tampoco estaba ella promoviendo actitudes tan importantes para el desarrollo económico como el espíritu de empresa, ni proporcionando la cantidad y calidad de competencia técnica necesaria para el desarrollo de las nuevas ocupaciones que surgen con el desarrollo". (Leyton: 1970, 69).

Es en virtud de este tipo de consideraciones que la Comisión de Planeamiento Integral de la Educación enuncia en 1967 los principios básicos de la Reforma para la educación media, en los términos siguientes: "Es una sentida aspiración entre nosotros la de democratizar nuestra educación media. Se desea crear igualdad de oportunidades educacionales... Esta igualdad de oportunidades debe materializarse en dos sentidos: cuantitativamente, es decir, dando acceso al sistema al mayor número posible de adolescentes, y cualitativamente, es decir, proporcionando iguales niveles de estudio a todos los educandos de la escuela media. La igualdad de niveles cualitativos es un factor que facilita y promueve la integración social y la movilidad dentro del sistema". (Revista de Educación $\mathrm{N}^{\circ} 3,1967$, p. 3 - 6). Desde un punto de vista práctico, además, a esta igualdad en el acceso, la permanencia y la calidad, se agrega otra medida de crácter democratizador muy básico, la flexibilización de las barreras que separan la educación técnica de la universitaria.

Sin embargo, todas estas consecuencias, indiscutiblemente democratizadoras se ven limitadas y hasta en parte contradichas por otras tantas orientaciones conceptuales de sentido contrario. Estas orientaciones aparecen, por ejemplo, en el discurso oficial sobre la educación superior. En una de las alocuciones del Presidente Frei sobre este tema podemos leer por ejemplo que "(los) sistemas selectivos que exigen las universidades para la admisión de alumnos no autorizan para decir que la educación superior constituye un privilegio, sino más bien un imperativo insoslayable de la ciencia y la tecnología modernas". (Revista de Educación, $N^{\circ} 5,1968,2-3$ ). Reservas similares frente a la democratización de este tramo de la educación aparecen, por ejemplo, en Juan Gómez Millas, esta vez respecto a la participación estudiantil en el gobierno universitario9.

Estas limitaciones en las ideas sobre una educación democrática aparecen también en las orientaciones de los líderes reformistas sobre las nuevas "carreras" o filieres educacionales que comunican a la rama técnico-profesional con el mercado de trabajo y a la educación científico-humanista con la Universidad. La opción entre la educación técnica y la científica humanista, a menudo determinada por razones económicas y que tienen que ver con pertenencia a grupos sociales determinados, es planteada en muchos documentos en términos de una opción, o de preferencias estrictamente individuales. Así por ejemplo, en el discurso de inauguración de la Reforma, dice el Ministro Gómez Millas que al término "de los 8 años de estudios generales comunes, después del período de orientación, los alumnos que deseen proseguir estudios se distribuirán, de acuerdo con sus intereses y capacidades, en los diversos tipos de escuela de enseñanza media en un ciclo de cuatro años". (Gómez Millas, 1969, 22).

\footnotetext{
"Ver sobre este punto de J.Gómez Millas "Sobre reformas universitarias y cogobierno" en Revista de Educación $x^{\circ} 2$, noviembre de 1967, p. 2.
} 
Como lo vemos y a pesar de esta apertura bastante profunda al tema de las relaciones entre educación y democratización, en definitiva los temas más básicos de esta relación, que tienen que ver con las clases sociales son velados por este discurso de la selección y la opción personal, que es común a Gómez Millas y a los restantes coordinadores de la Reforma. Estos son precisamente los temas que van a estar en el centro de los enfoques basados en la reproducción de las relaciones sociales, que de alguna manera van a suceder a las teorías del desarrollo a partir de los años 1970.

\section{III}

$\mathrm{Al}$ concluir esta breve presentación parecen adecuadas algunas orientaciones evaluativas. Hemos indicado ya en el texto nuestras reservas sobre el sesgo economicista que le imponen a la preocupación por el tema educacional las teorías del crecimiento y el desarrollo económico. Como lo decíamos, si la educacion es inversión, entonces es una variable esencial del desarrollo económico. Pero la pregunta es ¿qué educación puede ser considerada como inversión? La respuesta es que la educación que es efectivamente inversión es la que se relaciona de una manera más o menos directa con la capacitación para el trabajo productivo. Desde las teorías de la modernización, a estos tipos de saber es posible agregar algunos otros: las ciencias naturales sobre todo, pero también las ciencias sociales. En esta nueva distribución del poder al interior de las formas del saber, el lugar de las humanidades y la filosofía, por ejemplo, es no sólo secundario sino que puede representar obstáculos positivos a la construcción de una sociedad moderna.

He subrayado también, en las páginas que enteceden, los límites de la concepción democrática que complementa a las concepciones del desarrollo y más en particular, a la Reforma de 1965. Se trata de una concepción básicamente elitista, que supone una ampliación del universo que compite por formar parte de las elites pero que de ningún modo cuestiona el hecho de que la sociedad tenga que dividirse necesariamente en elites y masas.

A partir de los trabajos de filósofos políticos contemporáneos como John Rawls, es posible cuestionar aún otras características del modelo elitista de la Reforma. En base a su Teoría de la Justicia, se puede sostener que una meritocracia como la que se desprende de los principios de la Reforma de 1965, carece de fundamentos normativos, si es así que su origen son los talentos y capacidades individuales. En rigor, recalca Rawls, las habilidades y talentos, en particular, no pueden ser la base de una sociedad justa porque tienen un origen completamente arbitrario en una "lotería natural". Menos aún puede ser esto así si los talentos y las capacidades resultaran ser dependientes de las condiciones sociales en que se desarrollan los primeros años de vida de los niños. Pocas razones hay, pues, para considerar legítima una selectividad social y cultural, si su fundamento son estos talentos.

Por último, a partir de la obra de Jacques Ranciere, es posible formular un cuestionamiento mucho más global y radical de las teorías del desarrollo en educación. La tesis de Ranciere, que este autor comparte con otras visiones, como las de Michael Walzer y Hannah Arend $t^{10}$ es que hay un contrasentido profundo en la pretensión de ligar funcio-

\footnotetext{
${ }^{10}$ Sobre este punto, aparte el texto de Ranciere puede consultarse, de Michael Walzer, Esferas de Justicia (México: Fondo de Cultura Económica,1993) y de Hannah Arendt, "The crisis of education" en Between past and future, (Harmondsworth, Middlessex: Penguin, 1983).
} 
nalmente la escuela a la producción, defendiendo al mismo tiempo el carácter democrático de una educación así funcionalizada. Para Ranciere, "(antes) de ser el lugar de la transmisión del saber, la escuela es una forma social, un espacio-tiempo sustraído al universo del trabajo productivo. Es en virtud de esta forma que hace iguales a quienes acoge. Democratizar la escuela es, de partida, generalizar este retiro. Una escuela democrática y orientada a la producción es necesariamente una forma contradictoria". (Ranciere: 1990, 80). Según este autor, mucho más que el instrumento de programación de una ciencia útil a la empresa, "la escuela es el lugar privilegiado de la negociación de la igualdad, portador de modelos de sociedad que ponen en crisis los modelos sociales arraigados en la "capacitación" para la vida productiva. Sus efectos de transformación social no podrían dejar de conformarse a su esencia: la puesta a distancia de la producción". (Ibid., 86). El fundamento de esta aseveración la encuentra Ranciere sobre todo en el hecho de que aquellos que le han tomado el gusto a la igualdad escolar están "virtualmente perdidos para un mundo de la producción que es de partida un mundo de la desigualdad y de la ausencia de ocio". (Ibid., 83).

Vemos así cómo, desde diferentes puntos de vista, teorías como las del dasarrollo son fundamentalmente deficitarias como contribuciones a la construcción de una sociedad democrática. Su sesgo economicista tiende a hacernos perder de vista que no es una opción la que lleva, por una parte, a la mayoría de los adolescentes de los sectores populares hacia una educación vocacional o técnica. Por otra parte estas concepciones eluden el hecho fundamental de que las áreas laborales, con las que la escuela está ligada, son fundamentalmente relaciones de poder y que una muy pronta subordinación de la forma escolar al mundo del trabajo, conlleva también una marginalización de los modos de vida igualitarios que son, a la inversa, una contribución fundamental de la escuela moderna a la democracia.

\section{REFERENCIAS}

ARNDT, H.. El desarrollo económico. La historia de una idea. Buenos Aires: REI, 1992).

Angell, Alan, Chile de Alessandri a Pinochet: En busca de la utopía (Santiago: Andrés Bello. 1993).

APTER. DAvid, Política de la modernización (Buenos Aires: Paidós, 1972).

Germani, Gino, Política y sociedad en una época de transición. De la sociedad tradicional a la sociedad de masas (Buenos Aires: Paidós, 1969).

Góngora, Mario, Ensayo histórico sobre la noción de Estado en Chile en los siglos XIX y XX, (Santiago: Editorial Universitaria, 1986).

LipSET, Seymour, Elites y desarrollo en Anérica Latina (Buenos Aires: Paidós, 1967).

Leyton, MARio, La experiencia chilena: La Reforma educacional 1965-1970 (Santiago: CPEIP, 1970).

Leyton, Mario y CARKOVIC, ANTONio, "La reforma de la enseñanza media y sus proyecciones", Revista de Educación, $\mathrm{N}^{\circ} 4$, marzo 1968.

PREBISCH. RAĹL, "Introducción" a la Conferencia sobre educación y desarrollo económico-social en América Latina, UNESCO, Santiago, 1962.

RANCIERE, JACQLES, “Ecole, production, égalité" en X. Renou (Ed.) L'ecole de la démocratie (Paris: Fondation Diderot, 1990).

SCHULtz, TheOdore, Valor económico de la educación (México: UTEHA, 1968).

SLPERINTENdencia de EduCACión Péblica, La superintendencia de educación y la reforma educacional, Santiago, 1969. Unesco, Los problemas y la estrategia del planeamiento de la edacación. (La experiencia de América Latina), (Paris: UNESCO, 1965).

Revista de Educación, Santiago, 1967-1970. 